\title{
OPEN An in vitro study on Staphylococcus schweitzeri virulence
}

\author{
Almut Grossmann ${ }^{1}$, Neele J. Froböse ${ }^{1}$, Alexander Mellmann ${ }^{1,2}$, Abraham S. Alabi ${ }^{3}$, \\ Frieder Schaumburg ${ }^{1,5 \bowtie}$ \& Silke Niemann ${ }^{1,4,5}$
}

Staphylococcus schweitzeri belongs to the Staphylococcus aureus-related complex and is mainly found in African wildlife; no infections in humans are reported yet. Hence, its medical importance is controversial. The aim of this work was to assess the virulence of $S$. schweitzeri in vitro. The capacity of African S. schweitzeri $(n=58)$ for invasion, intra- and extracellular cytotoxicity, phagolysosomal escape, coagulase activity, biofilm formation and host cell activation was compared with $S$. aureus representing the most common clonal complexes in Africa (CC15, CC121, CC152). Whole genome sequencing revealed that the $S$. schweitzeri isolates belonged to five geographical clusters. Isolates from humans were found in two different clades. S. schweitzeri and S. aureus showed a similar host cell invasion ( 0.9 vs. $1.2 \mathrm{CFU} /$ Vero cell), host cell activation (i.e. expression of pro-inflammatory cytokines, 4.1 vs. 1.7 normalized fold change in gene expression of $C C L 5 ; 7.3$ vs. 9.9 normalized fold change in gene expression of $I L 8, A 549$ cells) and intracellular cytotoxicity ( $31.5 \%$ vs. $25 \%$ cell death, A549 cells). The extracellular cytotoxicity ( $52.9 \%$ vs. $28.8 \%$ cell death, A549 cells) was higher for $S$. schweitzeri than for $S$. aureus. Nearly all tested $S$. schweitzeri $(n=18 / 20)$ were able to escape from phagolysosomes. In conclusion, some $S$. schweitzeri isolates display virulence phenotypes comparable to African S. aureus. S. schweitzeri might become an emerging zoonotic pathogen within the genus Staphylococcus.

Staphylococcus aureus, Staphylococcus argenteus and Staphylococcus schweitzeri form the S. aureus-related complex ${ }^{1}$. The virulence and clinical outcome of $S$. aureus and $S$. argenteus is comparable but the capacity of $S$. schweitzeri to cause disease in humans remains controversial since no clinical infection has been reported, yet ${ }^{2}$. S. schweitzeri differs from $S$. aureus in the peptidoglycan type; the Genome-wide average nucleotide identity is only $88.6 \%{ }^{1}$. In routine diagnostics, $S$. schweitzeri can be distinguished from $S$. aureus based on a nuc-PCR, NRPS-gene or by MALDI-TOF².

Staphylococcus schweitzeri is mainly found as a colonizing strain of the nasopharynx in Afrotropical wildlife, particularly in bats and primates, but can also be detected on fomites ${ }^{3,4}$. So far, only three cases of a nasopharyngeal S. schweitzeri colonization in humans were reported from rural Gabon and might have been acquired while handling bushmeat ${ }^{5,6}$. S. schweitzeri can carry almost the same virulence factors as described for the other members of the $S$. aureus-related complex such as enterotoxins, toxic shock syndrome toxin, exfoliative toxins, leukocidins, hemolysins, adhesins, autolysins, immune evasion factors, or polysaccharide capsules ${ }^{2}$. A first functional study revealed that the $S$. schweitzeri type strain FSA084T produces $\alpha$-hemolysin and is cytotoxic to human cell lines (HeLa, HT29) ${ }^{7}$. These preliminary results lead us to the hypothesis that the virulence of S. schweitzeri and S. aureus is equivalent. However, other functional aspects (e.g. invasion) of S. schweitzeri have not been studied, yet. Since virulence differs between isolates of various genetic backgrounds in in vitro studies, a representative strain collection of $S$. schweitzeri is needed. In addition, no clinical samples from humans in sub-Saharan Africa were so far screened systematically for $S$. schweitzeri to detect spill-over events from wildlife to humans.

Therefore, the aims of this study were (1) to test the virulence of $S$. schweitzeri in a wide range of in vitro assays on the largest $S$. schweitzeri collection published so far and (2) to screen systematically clinical samples from humans living in an area where $S$. schweitzeri is reported frequently in wildlife.

\footnotetext{
${ }^{1}$ Institute of Medical Microbiology, University Hospital Münster, Münster, Germany. ${ }^{2}$ Institute for Hygiene, University Hospital Münster, Münster, Germany. ${ }^{3}$ Centre de Recherches Médicales de Lambaréné, Lambaréné, Gabon. ${ }^{4}$ Section of Medical and Geographical Infectiology, University Hospital Münster, Münster, Germany. ${ }^{5}$ These authors contributed equally: Frieder Schaumburg and Silke Niemann. ${ }^{\square}$ email: frieder.schaumburg@ukmuenster.de
} 

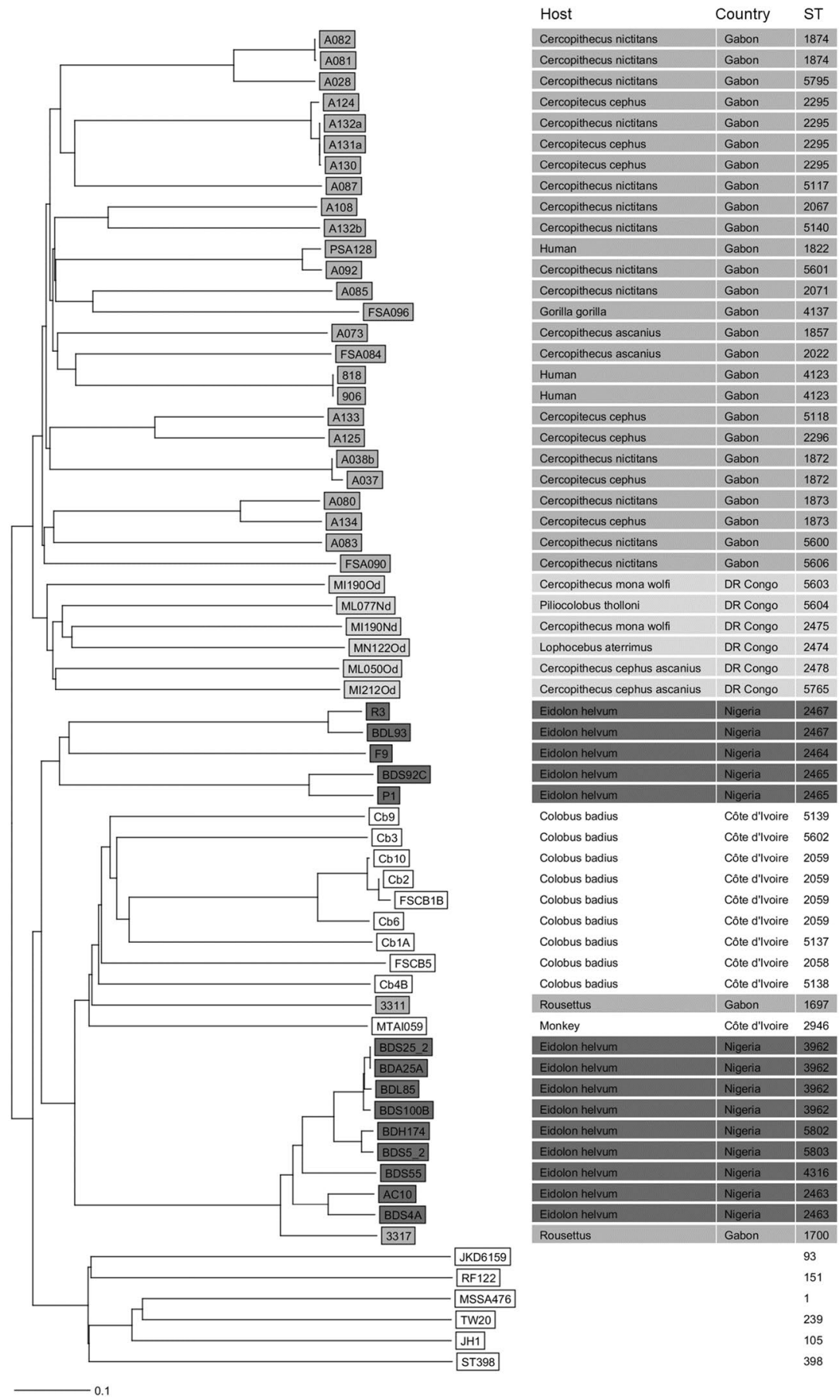

Figure 1. Neighbour-joining tree of Staphylococcus schweitzeri. The genomes of S. schweitzeri $(\mathrm{n}=58)$ from African wildlife and humans in Côte d'Ivoire, Democratic Republic of the Congo, Nigeria and Gabon were sequenced and the phylogenetic tree was constructed based on the up to 1861 targets of the $S$. aureus cgMLST scheme $^{29}$. MLST ST, host and country of origin are indicated for each isolate. The colour-code represents the country of origin. S. aureus strains JKD6159, RF122, ST398, JH1, MSSA476 and TW20 were selected for comparison ${ }^{1}$.

\section{Results}

Phylogeny. A total of 58 isolates from four African countries were included in the study and were confirmed to be $S$. schweitzeri as all clustered in a neighbor-joining tree based on the cgMLST scheme with the $S$. schweitzeri type strain (FSA084 = DSM 28300) separately from S. aureus standard strains (Fig. 1). S. schweitzeri isolates were grouped in five geographical clusters including two from Nigeria. The three $S$. schweitzeri from humans grouped in two separate clades from Gabon. The most closely related isolates of the human isolates were detected in monkeys (C. nictitans, C. ascanius). 
S. schweitzeri screening. To test if S. schweitzeri can cause clinical infection that may have gone unnoticed, we screened clinical isolates $(\mathrm{n}=156)$ provisionally identified as $S$. aureus for characteristics of $S$. schweitzeri (i.e. negative nuc-PCR, 340 bp NRPS). These isolates are from the Lambaréné region in Gabon, where the only human carriers of $S$. schweitzeri were found so far. In total, the majority of isolates were from wound swabs $(\mathrm{n}=43,27.5 \%)$, followed by abscesses $(\mathrm{n}=24,15.4 \%)$ and skin and soft tissue infections $(\mathrm{n}=22,14.1 \%)$ and others $(\mathrm{n}=67,43.0 \%)$. All isolates were confirmed to be $S$. aureus (i.e. positive nuc-PCR, 160 bp NRPS). Thus, $S$. schweitzeri was not detected in human cases of infection.

Coagulase assay. The majority of $S$. schweitzeri isolates was able to coagulate rabbit plasma $(\mathrm{n}=57,98 \%)$ followed by human plasma $(n=55,95 \%)$ and chimpanzee plasma $(n=39,67 \%) .39$ isolates $(67 \%)$ coagulated all three different plasma (Figure S1).

Growth curves. Staphylococcus schweitzeri appeared to grow better than African S. aureus isolates at $34^{\circ} \mathrm{C}$ and $40^{\circ} \mathrm{C}$, the corresponding body temperatures of bats and monkey, respectively (Fig. 2). A further statistical comparison of the growth curves using a two-way repeated measures ANOVA was not possible due to inhomogeneity of data on account of numerous extreme outliers in both groups (Fig. 2).

Invasion of S. schweitzeri in epithelial cells of human and monkey origin. The ability to invade human epithelial lung cells (A549) and monkey kidney cells (Vero) was compared between S. schweitzeri and African S. aureus (Fig. 3A). The mean number of colony forming units (CFU) in Vero cells was 0.9 CFU/cells (S. schweitzeri) and 1.2 CFU/cells (African S. aureus). For A549 cells, S. schweitzeri was detected more often intracellularly than $S$. aureus (2.5 CFU/cells vs. $1.3 \mathrm{CFU} /$ cells). However, if the ability to invade Vero cells and A549 cells is equivalent or statistically different in S. schweitzeri and S. aureus remained undetermined in the two one-sided test (TOST) procedure (Equivalence Test: $\mathrm{p}>0.9$, null hypothesis significance tests [NHST]: $\mathrm{p}>0.1$ ). While the African S. aureus seem to invade Vero and A549 cells in comparable numbers, S. schweitzeri was more often detected intracellularly in A549 cells compared to Vero cells (2.5 CFU/cells vs. 0.9 CFU/cells).

The ability to invade host cells varied markedly among the different $S$. schweitzeri isolates for A549 cells (range 0.2-7.9 CFU/cells) and Vero cells (range 0.1-6.9 CFU/cells, Figure S2). The majority of S. schweitzeri invaded human epithelial cells (A549) better than monkey kidney cells (Vero cells). The invasion of certain S. schweitzeri isolates was similar to African S. aureus strains.

Intracellular cytotoxicity. The cytotoxicity (i.e. \% of dead cells after infection) was noticeably similar for S. schweitzeri and African S. aureus in Vero cells (14.7\% vs. $14.2 \%)$ and A549 cells (31.5\% vs. $25 \%)$ but both the equivalence test and NHST were not statistically significant for Vero cells $(p=0.5$ and $p=0.8$, respectively) and A549 cells ( $\mathrm{p}=0.3$ and $\mathrm{p}=0.2$, respectively). Therefore, it remains undetermined, if the intracellular cytotoxicity is equivalent or statistically different in S. schweitzeri and S. aureus.

Furthermore, the A549 cells were significantly more susceptible to intracellular cytotoxicity caused by $S$. schweitzeri than Vero cells $(31.5 \%$ vs $14.7 \%$ p $<0.001$, student's t-test, Fig. 3B). Noteworthy, intracellular cytotoxicity differed markedly among the $58 \mathrm{~S}$. schweitzeri isolates for A549 cells (range 8.1-58.5\%) and Vero cells (range $8.3-39.0 \%$, Figure S3).

Phagolysosomal escape. Almost all randomly selected S. schweitzeri (18/20) were able to escape from phagolysosomes (mean: 1.82 bacteria/cell, Figure S4). The S. aureus strain 6850 and USA300 showed a phagosomal escape with a mean of 1.78 bacteria/cell and 1.8 bacteria/cell, respectively (Figure S4). However, we detected a large variance within the different $S$. schweitzeri isolates (range 0.1-3.5 escaped bacteria/cells, Figure S4).

Expression of inflammation-related genes. The inflammatory response of A549 cells to an infection with 20 randomly selected $S$. schweitzeri and three African $S$. aureus isolates (one strain each of the three most common clonal MLST complexes in sub-Saharan Africa) was determined by the expression of IL8 and CCL5. After $8 \mathrm{~h}$, the expression levels (in normalized fold change of gene expression) were $4.1 \pm 2.3$ (CCL5) and 7.3 \pm 4.0 (IL8) in cells infected with $S$. schweitzeri and $1.7 \pm 0.5$ (CCL5) and $9.9 \pm 5.9$ in cells infected with $S$. aureus. After the TOST procedure, it remained undetermined, if differences in the expression level of CCL5 and IL8 were different $(\mathrm{p}>0.1)$ or equivalent $(\mathrm{p}>0.4)$ after $8 \mathrm{~h}$.

The expression of IL8 was not different after $24 \mathrm{~h}$ in A549 cells infected with S. schweitzeri or S. aureus. In contrast, CCL5 expression after $24 \mathrm{~h}$ was significantly higher (and not equivalent) in A549 cells infected with S. schweitzeri compared to A549 cells infected with African S. aureus (8.3 vs 1.8 normalized fold expression, $\mathrm{p}=0.02$, Figure S5). Similar to invasion, intracellular and extracellular cytotoxicity, the gene expression induced by $S$. schweitzeri infection varied among the 20 S. schweitzeri markedly (Figure S6).

Biofilm. The thermostable nuclease (Nuc) plays a critical role in biofilm formation ${ }^{8}$. Since several polymorphisms of nuc are described for S. schweitzeri, we compared the biofilm formation between S. schweitzeri and S. aureus $^{9}$. The ability to form biofilm was noticeably higher in S. schweitzeri than in African S. aureus (mean OD 0.16 vs. 0.09 ). Based on the TOST procedure it remains undetermined if the biofilm formation of $S$. schweitzeri vs. $S$. aureus is equivalent $(\mathrm{p}=0.3)$ or statistically significantly different $(\mathrm{p}=0.2)$. Both species were weak biofilm producers compared to the positive control Staphylococcus epidermidis RP62A (OD 0.42). S. schweitzeri showed a higher ability to form biofilm than the negative control S. carnosus TM300 (OD 0.08, p=0.04, Fig. 3C). Genes 

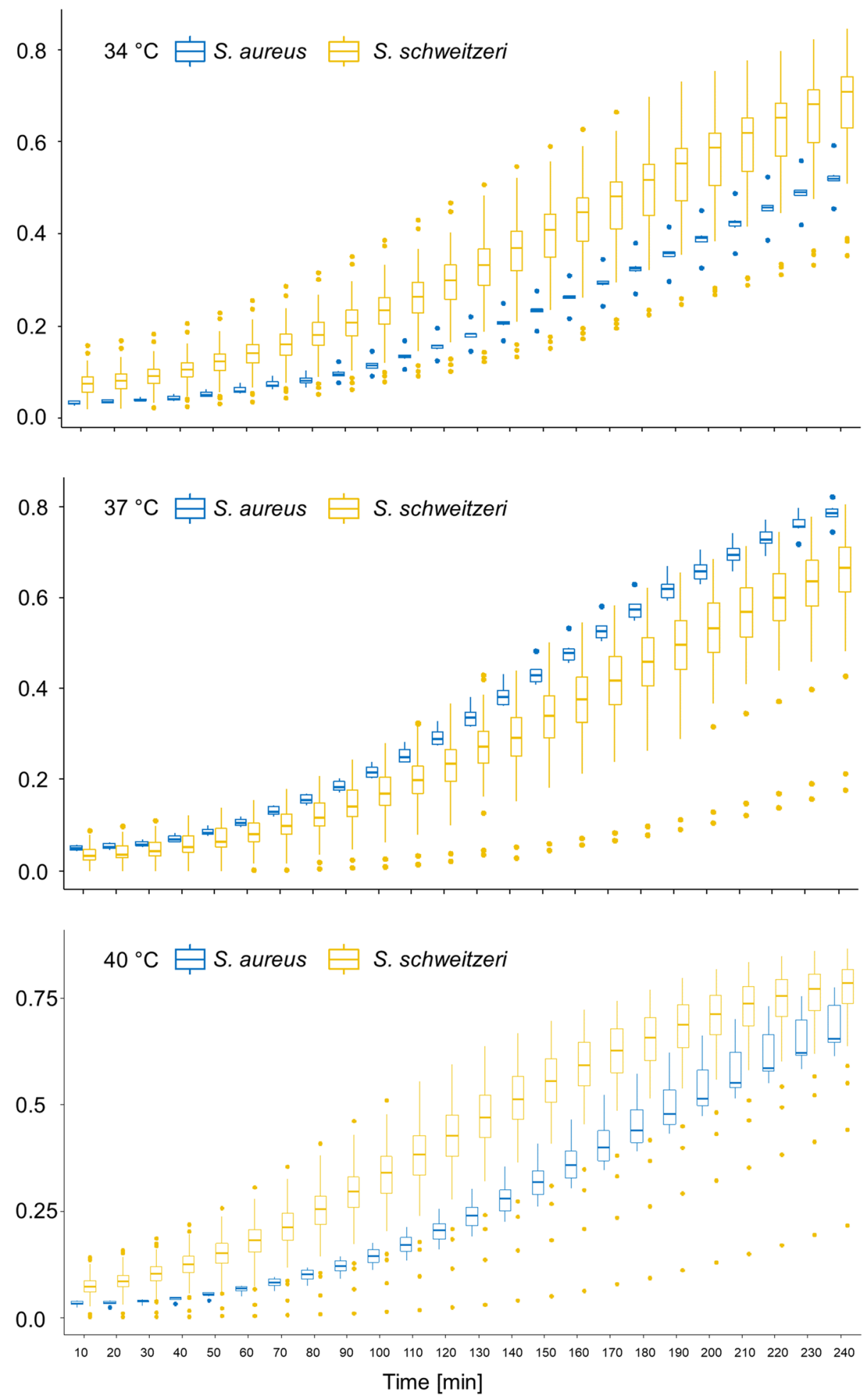

Figure 2. Growth curve of Staphylococcus schweitzeri and African Staphylococcus aureus at different temperatures. The growth of $S$. schweitzeri $(\mathrm{n}=58$, yellow boxplots) and a representative selection of African S. aureus ( $\mathrm{n}=6$, blue boxplots) was photometrically measures after intervals of $10 \mathrm{~min}$ for $4 \mathrm{~h}$. Boxplots (incl. outliers) of optical density (OD) are shown for each species and time points. S. schweitzeri grew faster at $34^{\circ} \mathrm{C}$ and $40{ }^{\circ} \mathrm{C}$ than $S$. aureus comparator isolates. 

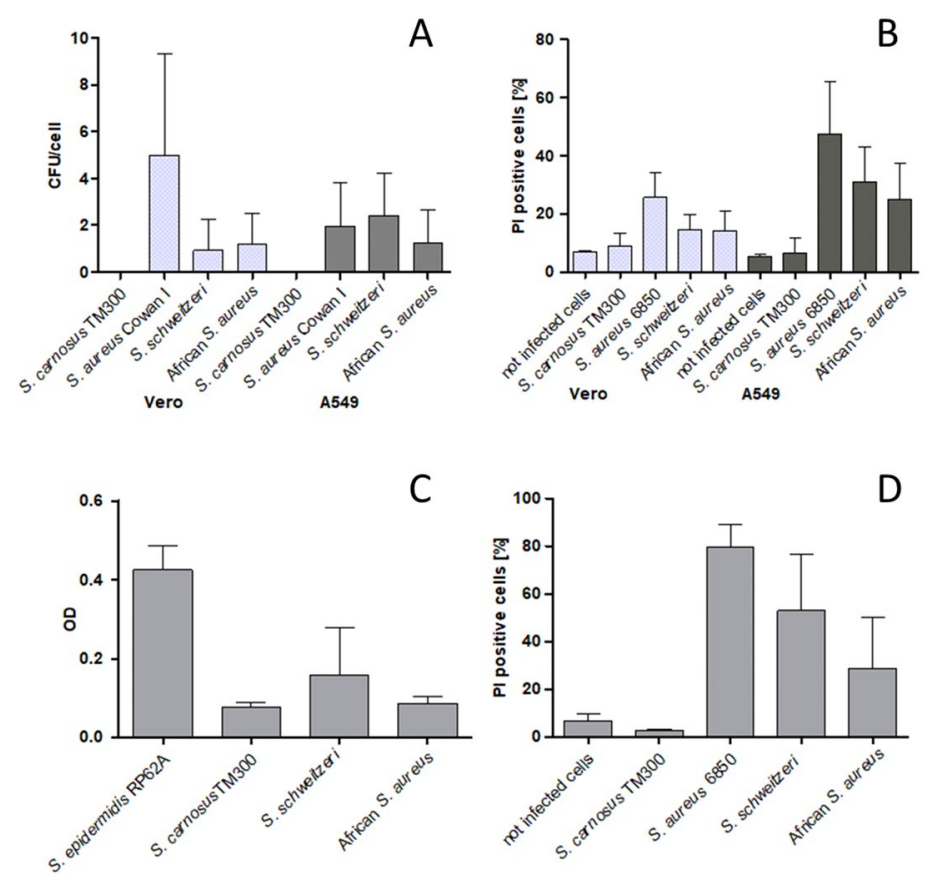

Figure 3. Comparison of the in vitro virulence of Staphylococcus schweitzeri and representative African Staphylococcus aureus. The results were merged for S. schweitzeri $(\mathrm{n}=58)$ and African S. aureus $(\mathrm{n}=6)$ and displayed as the mean $( \pm$ SD) for each of the two groups. (A) Cellular invasion in Vero and A549 cells. The highly invasive strain S. aureus Cowan I served as positive and the low invasive S. carnosus strain TM300 as negative control. (B) Intracellular cytotoxicity after infection of Vero and A549 cells. The highly cytotoxic S. aureus strain 6850 served as positive and the S. carnosus strain TM300 as negative control. (C) Biofilm formation. The biofilm production was measured photometrically and bars indicated mean OD $( \pm S D)$. (D) Extracellular cytotoxicity. A549 cells were exposed to secreted toxins in supernatants of overnight cultures. The mean cytotoxicity (proportion of dead cells, \pm SD) of $S$. schweitzeri was significantly stronger than for African $S$. aureus $(\mathrm{p}=0.02)$.

associated with high formation of biofilm (i.e. bap and icaA), were not detectable in S. schweitzeri using whole genome sequencing. African S. aureus were tested positive for icaA, but also negative for bap.

Extracellular cytotoxicity. As intracellular effects are only one aspect of virulence, we also investigated the cytotoxicity of secreted products of $S$. schweitzeri on A549 cells. The supernatant of overnight cultures of $S$. schweitzeri was significantly more cytotoxic compared to the African S. aureus strains (52.9\% vs. 28.8\%, Fig. 3D) in the TOST procedure (equivalence test: $\mathrm{p}=0.7$, NHST: $\mathrm{p}=0.02$ ). Supernatants of the negative control S. carnosus TM300 had no cytotoxic effect; supernatants of the positive control S. aureus 6850, on the other hand, had a very high cytotoxic effect. Similar to intracellular cytotoxicity, extracellular cytotoxicity differed strongly among the 58 S. schweitzeri isolates (range 6.4-89\%, Figure S7).

\section{Discussion}

We compared the in vitro virulence of $S$. schweitzeri with a selection of African S. aureus lineages and found that certain isolates of $S$. schweitzeri share similar virulence traits with African $S$. aureus strains.

The five phylogenetic clusters of $S$. schweitzeri rather correspond to the geographical origin than the host (Fig. 1). A definite delineation of geographical vs. host-related clusters is confounded by the fact that some host species are only found in one geographical area (e.g. E. helvum in Nigeria, C. badius in Côte d'Ivoire). However, geographical clusters of $S$. schweitzeri were also reported based on the nuc sequences ${ }^{9}$.

Staphylococcus schweitzeri was not detected in clinical samples in our study. Unlike colonization, there is no evidence that $S$. schweitzeri can cause infection in humans so far. However, the sole absence of $S$. schweitzeri in human infection should not be considered as a point neither for nor against its virulence. It might only be a snapshot of a temporal process. A cross-species transmission from wildlife to humans still remains possible since wildlife, particularly great apes or bats, are considered sentinels for the anticipation or early detection of zoonotic diseases in humans ${ }^{10}$.

While a coagulase activity for rabbit and human plasma was expected, the low numbers of isolates coagulating chimpanzee plasma $(n=39,67 \%)$ was surprising. This could suggest a poorer adaptation of $S$. schweitzeri to great apes ${ }^{11}$. However, since great apes are the closest relatives to humans, one would expect a similar coagulase activity for plasma from humans and chimpanzees. A caveat for this comparison is that chimpanzee plasma was 
haemolytic (as observed by the naked eye through the change in plasma colour) indicative for a release of cellular molecules that interfere with coagulation (e.g. proteases, ADP) and only tested in monoplicates ${ }^{12}$.

Staphylococcus schweitzeri appeared to grow better at $34^{\circ} \mathrm{C}$ and $40^{\circ} \mathrm{C}$ than S. aureus (Fig. 2) suggesting that cell division of $S$. schweitzeri is more adapted to the mean body temperature of bats $\left(34^{\circ} \mathrm{C}\right)$ and monkeys $\left(40^{\circ} \mathrm{C}\right)$ than to humans ${ }^{13}$. This is in line with the observation, that $S$. schweitzeri is mostly found in bats and monkeys ${ }^{2}$.

Most of the S. schweitzeri strains invade both A549 and Vero cells (Fig. 3A, Figure S2). The invasion of $S$. aureus highly depends on the host cell type and the expression of virulence factors, which varies between strains $^{14}$. One of the main factors for the uptake of $S$. aureus in host cells is the expression of fibronectin binding proteins $\mathrm{A}$ and $\mathrm{B}(\mathrm{FnBPA}, \mathrm{FnBPB})^{15}$. The genes for one or both of these adhesins are also present in the genome of most of the S. schweitzeri isolates studied (Table S3). In contrast, all isolates lacked the gene for extracellular adherence protein Eap (also called Map), which, in addition to its adhesive and immunomodulatory properties, can also contribute to the invasion of $S$. aureus in host cells in the absence of FnBPs ${ }^{16}$. Interestingly, also in African S. aureus isolates only fragments and not the full map gene was detected ${ }^{17}$.

Staphylococcus schweitzeri can translocate from the phagolysosome into the cytoplasm after phagocytosis (Figure S3). The ability to escape from phagolysosome and to multiply in the cytoplasma of non-professional phagocytes eventually leading to cytolysis is now shown not only for $S$. aureus but also for $S$. schweitzeri ${ }^{18,19}$. The very low number of escaped bacteria/cell of some isolates (BDS92c, FSA096, P1) can possibly be attributed to the fact that these strains are only taken up to a small extent by the host cells (Figure S2).

The invasion of $S$. aureus into host cells triggers the release of pro-inflammatory cytokines ${ }^{14}$. This host defence can also exacerbate the course of the disease ${ }^{20}$. In the present study, we investigated the mRNA levels of CCL5and IL8 as examples for pro-inflammatory cytokines. CCL5 (RANTES) is chemotactic for T-cells, eosinophils, and basophils, and plays an active role in recruiting leukocytes into inflammatory sites. IL-8 (CXCL8) is a neutrophil chemoattractant that plays a central role in innate immunity to bacterial infection. Blood cells and many types of tissues produce IL-8. We showed that after infection with $S$. schweitzeri the host cells respond with a defense reaction, and the increase in cytokine expression is maintained for $24 \mathrm{~h}$ (Figure S6).

Staphylococcus schweitzeri produced more biofilm than the African S. aureus but the level of biofilm production was still markedly lower compared to the positive control S. epidermidis RP62A (Fig. 3C). Biofilm formation is regulated by numerous virulence factors such as the thermostable nuclease Nuc. Although the primary structure of Nuc from S. schweitzeri $\left(n u c_{M}\right.$ ) and S. aureus (nuc1) differ (identity 78.1-80.4\%, similarity 92.4-94.1\%), the nuclease activities are identical which is in line with a similar biofilm formation ${ }^{9}$. The weak biofilm formation by $S$. schweitzeri could be attributed to the lack of the biofilm producing genes bap or icaA. As bap was not detected in both S. schweitzeri and the African S. aureus, this could explain the weak biofilm formation. The African $S$. aureus was tested positive for icaA, but gene expression was not analysed.

Our study has some limitations. First, the African S. aureus controls showed low virulence compared to the positive controls in all assays. This is most likely due to the small sample size of this control group. Second, we saw differences in the cytotoxic effect of bacteria on human epithelial cells compared to monkey kidney cells. However, we cannot conclude that this is due to species adaptation as the cells are from different tissues (i.e. lung vs. kidney). Third, we applied in vitro assays, which give a hint but do not allow drawing definite conclusions about virulence in vivo. Further studies, also in animal models, are necessary to predict more accurately whether S. schweitzeri is pathogenic in humans or not. Fourth, although it is possible, our phylogenetic analysis does not allow for conclusions on transmission of $S$. schweitzeri between animals and humans. Further, particularly prospective observations are need to address transmission events. Fifth, due to the large sample size we did not adjust for the starting inoculum of the growth curves and used one colony of an overnight culture instead which might have affected the increase in growth. However, the small interquartile range at the first measuring point suggests that our approach most likely does not produce misleading results (Fig. 2).

In conclusion, the variance of $S$. schweitzeri in the applied virulence assays is large and certain isolates display virulence phenotypes comparable to African S. aureus isolates in the applied in vitro assays; however, clinical infections were not detected. S. schweitzeri might become an emerging zoonotic pathogen, if a cross-species transmission from African wildlife sustainably occurs.

\section{Materials and methods}

Ethical considerations. All clinical isolates from humans derived from the routine laboratory of the Albert Schweitzer Hospital in Lambaréné, Gabon. Informed consent was waived by the Institutional Ethics Committee of the "Centre de Recherches Médicales de Lambaréné [CERMEL]") ${ }^{21}$. All methods were carried out in accordance with relevant guidelines and regulations of Gabon (https://cermel.org/ethicscommittee.php). All experimental protocols were approved by the Medical Faculty, Westfälische Wilhelms-Universität Münster, Germany (SC121720).

Bacterial isolates. Staphylococcus schweitzeri isolates $(\mathrm{n}=58)$ were collected in Gabon $(\mathrm{n}=28)$, Nigeria $(n=14)$, Côte d'Ivoire $(n=10)$ and the Democratic Republic of the Congo $(n=6)$ between 2007 and 2017 (Table S1). They derived from monkeys $(n=38)$, bats $(n=16)$, humans $(n=3)$ and gorilla $(n=1)^{4-6,22-24}$.

The hypothesis of the study was that the virulence of $S$. schweitzeri and $S$. aureus is equivalent. Since $S$. schweitzeri is only found in Africa, we selected the most common African S. aureus lineages for reference and to control for a geographical bias. For that purpose, a total of six $S$. aureus reference strains from infection and colonization were selected from the three most common MLST clonal complexes (CC) in sub-Saharan Africa $(\mathrm{CC} 15, \mathrm{CC} 121, \mathrm{CC} 152)^{17,25}$. These six reference strains are part of a strain collection from the Democratic Republic of the Congo but represent major sub-Saharan African clones ${ }^{25}$. 
In preparation for the infection experiments bacteria from overnight culture in Tryptic Soy Broth (TSB) (shaking conditions, $37^{\circ} \mathrm{C}$ ) were adjusted to $\mathrm{OD}_{578} 0.1$ in TSB. After $3 \mathrm{~h}$ of growth under the same conditions as before, the bacteria were adjusted to $\mathrm{OD}_{578} 1$, and stored in aliquots at $-20^{\circ} \mathrm{C}$ until use. From a previously frozen aliquot the number of colony forming units (CFU) was determined after serial dilutions on blood agar of the bacterial suspensions and overnight incubation at $37^{\circ} \mathrm{C}$.

To test whether $S$. schweitzeri caused infections that may have gone unnoticed, clinical isolates $(\mathrm{n}=156)$ provisionally identified as $S$. aureus (catalase- and coagulase Gram-positive cocci) at the Albert Schweitzer Hospital, Lambaréné, Gabon (2010-2016) were screened for $n u c^{26}$ and the $340 \mathrm{bp}$-fragment of NRPS ${ }^{1,27}$. S. schweitzeri was defined by a negative $n u c$-PCR and the detection of the $340 \mathrm{bp}$-fragment of NRPS in catalase- and coagulase Gram-positive cocci².

Cell culture. We aimed to analyse the virulence using both human and monkey derived cell lines as $S$. schweitzeri is mainly found in monkeys and the $S$. aureus reference strains were from human origin. This selection was based on the assumption, that the two species were differently adapted to cell lines from different hosts. For that purpose, A549 cells (ACC 107, DSMZ GmbH, Braunschweig, Germany), a human alveolar epithelial cell line and Vero cells, a kidney epithelial cell line from monkeys were used (Vero cells were a gift from S. Ludwig, Institute of Molecular Virology, University of Münster). Cells were cultured in cell culture flasks (CELLSTAR, tissue culture-treated surface, Greiner Bio-One, Frickenhausen, Germany) in Dulbecco's modified Eagle medium (DMEM, Biochrom, Berlin, Germany), supplemented with 10\% fetal calf serum (FCS, Biochrom, Berlin, Germany) at $37^{\circ} \mathrm{C}$ and $5 \%$ carbon dioxide. For the infection experiments, A549 cells from passage 2 to passage 50 after defrosting from storage and Vero cells from passage 2 to 25 were used. Regular control experiments with well characterized $S$. aureus strains were used to check that cells from higher passages still showed the same behaviour as cells from the lower ones.

The cells were seeded at 40,000 cells $/ \mathrm{cm}^{2}$ in 12 well plates (Corning,Costar, tissue culture-treated surface, Wiesbaden, Germany) 2-3 days before the experiment and were used at $90-100 \%$ confluence. To quantify the cell number, cells were seeded in an additional well in the tissue culture plate. On the day of the experiment, these cells were detached with trypsin-EDTA and the cell number was determined using an automated cell counter (TC20, Bio-Rad, Feldkirchen, Germany).

Whole genome sequencing. Available S. schweitzeri sequences were retrieved from GenBank (accession nos. ERS140147, ERS140266, ERS140239, ERS140159, ERS140162, ERS140167) ${ }^{1}$. Sequences of African S. aureus reference strains were obtained from the European Nucleotide Archive (PRJEB15192) ${ }^{25}$. All other genomes were sequenced on a Illumina MiSeq platform (Illumina Inc., San Diego, USA) aiming for an at least 75-fold coverage $^{28}$. Quality trimming and de novo assembly using the Velvet assembler was done with SeqSphere+ (version 5.9.0; Ridom GmbH, Münster, Germany) $)^{28}$.

Genomes were screened for MLST sequence types (ST) and 98 different virulence factors as described elsewhere ${ }^{25}$. A neighbor-joining tree was constructed based on up to the 1,861 genes of the $S$. aureus core genome (cg)MLST scheme ${ }^{29}$. New reads were deposited at the European Nucleotide Archive (accession no: PRJEB35847).

Coagulase assay. Plasma from rabbits (bioMérieux, Marcy l'Etoile, France), humans (pooled from 10 different human samples, blood bank of the University Hospital Münster) and chimpanzees (pooled from seven chimpanzee samples, Allwetterzoo Münster, Münster, Germany, originally taken for veterinary purposes) were used to test the coagulase activity of $S$. schweitzeri towards different species (in triplicates). Due to the little volume of chimpanzee plasma, the test was performed only once. Bacterial cells from overnight cultures $\left(1 \times 10^{8}\right)$ were suspended in $300 \mu \mathrm{l}$ of the respective plasma and incubated for $4 \mathrm{~h}$ at $37^{\circ} \mathrm{C}$. A coherent clot when tilting the tubes was considered a positive result ${ }^{11}$. S. aureus ATCC 29213 and S. epidermidis ATCC 12228 were positive and negative controls, respectively.

Growth curves. Growth curves of S. schweitzeri $(\mathrm{n}=58)$ were photometrically measured ( $578 \mathrm{~nm}$, Bio-Rad, Hercules, CA, USA) in triplicates every ten minutes for $4 \mathrm{~h}$ at $34^{\circ} \mathrm{C}, 37^{\circ} \mathrm{C}$ and $40{ }^{\circ} \mathrm{C}$ to assess an adaptation to different body temperatures of the different hosts (bats $34.8-37^{\circ} \mathrm{C}$, humans $37^{\circ} \mathrm{C}$, monkeys $\left.40^{\circ} \mathrm{C}\right)^{13}$. For this, one colony of overnight cultures was suspended in $200 \mu \mathrm{l} \mathrm{TSB}$ and incubated in 96-well plates (flat bottom). Wells were shaken briefly before each measurement.

Invasion assay. A549 and Vero cells were infected with a multiplicity of infection of 50 (MOI50) in invasion medium (DMEM, 10\% human serum albumin, $10 \mathrm{mmol} / \mathrm{l}$ HEPES, Merck, Berlin, Germany) and incubated for $3 \mathrm{~h}$ at $37^{\circ} \mathrm{C}$. The highly invasive strain S. aureus Cowan I served as positive and the low invasive S. carnosus strain TM300 as negative control ${ }^{14,15}$. After washing, extracellular bacteria were eliminated by lysostaphin (20 mg/ml, WAK-Chemie Medical, Steinbach, Germany) for $30 \mathrm{~min}$. Cells were detached by EDTA-trypsin (Biochrom, Berlin, Germany). The number of cells was determined using an automated cell counter (BIO-RAD, TC20). Immediately afterwards, the cells were centrifuged and the pellet was taken up in ice-cold water for lysis of the cell. To determine the CFU, serial dilutions of the cell lysates were cultured overnight on Columbia blood agar at $37^{\circ} \mathrm{C}$. Invasion was expressed as the mean number of CFU per host cell.

Intracellular cytotoxicity. The infection of Vero and A549 cells was performed as for invasion assays. The highly cytotoxic S. aureus strain 6850 served as positive and the S. carnosus strain TM300 as negative control ${ }^{14}$. Extracellular bacteria were removed by lysostaphin $3 \mathrm{~h}$ post infection. After overnight incubation (in DMEM, 
10\% FCS, Penicillin, Streptomycin, Merck, Berlin, Germany), cells were washed with PBS and adherent cells were detached with EDTA-trypsin. Supernatant, PBS from the washing step and detached cells from one well were collected in one tube, so that cells already detached by cell damage or cell death were included in the analysis. Dead host cells were stained with propidium iodide (PI, PD Pharmingen, Heidelberg, Germany) and analysed by flow cytometry. Cytotoxicity was expressed as the proportion of dead (i.e. \% of PI-positive) cells.

Phagolysosomal escape. Twenty $S$. schweitzeri isolates were randomly selected (https://www.randomizer .org/) to analyse the phagolysosomal escape ${ }^{14}$. Briefly, A549 cells were used which express the escape marker YFP-CWT in the cytoplasm. YFP-CWT is recruited to the peptidoglycan of the bacterial cell wall upon rupture of the phagolysosomal membrane barrier ${ }^{30}$. Subsequently, fluorescent rings around the bacteria become visible and allow the quantification of bacteria that have escaped into the cytoplasm. A549 YFP-CWT cells were infected in imaging dishes with cover glass bottom (MoBiTec, Göttingen, Germany) with S. schweitzeri for $1 \mathrm{~h}$, followed by lysostaphin treatment and adding of fresh culture medium. After $2.5 \mathrm{~h}$, cells were analysed by fluorescence microscopy (Axio Observer.Z1, Carl Zeiss, Jena, Germany, Zeiss filter set 38 HE, 100x/NA 1.3 plan-neofluar objective). Pictures were documented with an AxioCam MRm camera and processed using Zeiss AxioVision. Ten fields of view were analysed for each isolate (Figure S8) and the phagolysosomal escape was expressed as the mean number $( \pm S D)$ of escaped bacteria per cell from three independent experiments. We defined that we do not consider an escaped bacteria/cell ratio $<0.5$ as a phagolysosomal escape.

Quantitative real-time-polymerase chain reaction (qRT-PCR). qRT-PCR was used to analyse the gene-expression of inflammation-related CCL5 and IL8 to determine the activation of A549 host cells after infection with 20 randomly selected $S$. schweitzeri ${ }^{14,31}$ and 3 African S. aureus isolates from colonisation (one strain each of the three most common clonal MLST complexes in sub-Saharan Africa). Infection of A549 with S. aureus 6850 served as a control.

Infection was performed as described for intracellular cytotoxicity. At 8 and 24 h post infection, cells were detached with EDTA-trypsin, centrifuged, suspended in RNAprotect (QIAGEN, Hilden, Germany) and stored at $-20^{\circ} \mathrm{C}$. After RNA extraction (RNeasy Mini kit, QIAGEN), the concentration of total RNA was determined using a Nanophotometer (Implen, Munich, Germany) and adjusted to $1000 \mathrm{ng}$. RNA quality was assessed by determining the A260/A280 ratio. This ratio was between 1.7 and 2, with most samples showing a ratio around 1.9. cDNA was synthetized (Quantitect reverse transcription kit, QIAGEN) following the manufacturer's recommendations and stored at $-80^{\circ} \mathrm{C}$.

Real-time amplification was done with the iQ SYBR Green Supermix (Bio-Rad) and specific primers (GAPDH, $B 2 M, C C L 5, I L 8$, Table S2) on an iCycler iQ real-time PCR-system (Bio-Rad), adding $50 \mathrm{ng}$ cDNA to the reaction. The reaction mixtures were incubated for $15 \mathrm{~min}$ at $95^{\circ} \mathrm{C}$ followed by 40 amplification cycles $\left(15 \mathrm{~s}\right.$ at $95^{\circ} \mathrm{C}, 30 \mathrm{~s}$ at $55^{\circ} \mathrm{C}, 30 \mathrm{~s}$ at $72^{\circ} \mathrm{C}$ ). iQ5 software was used to calculate PCR efficiencies, melting-curve analysis, and expression rates. $B 2 M$ and GAPDH were used as endogenous controls to normalize expression levels ${ }^{32}$. The reference genes were chosen based on our previous study ${ }^{14}$. Data are presented as normalized fold change in expression compared to controls (non-infected cells) using the $\Delta \Delta \mathrm{Ct}$ method. S. aureus strain 6850 was used as positive control.

Biofilm formation. Overnight BHI-cultures were diluted (200-fold in BHI, including $0.25 \%$ glucose) in 96-well plates (flat bottom, Greiner bio-one, Frickenhausen, Germany) and incubated overnight at $37^{\circ} \mathrm{C}$. Cultures were washed twice (PBS) and stained with $1 \%$ crystal violet (Labochem international, Heidelberg, Germany) for $15 \mathrm{~min}$. After washing (PBS, three times), the biofilm was dissolved in $100 \mu$ l ethanol-acetone (80:20) and the biofilm mass was measured photometrically $(655 \mathrm{~nm})$ in a microtiter plate reader (Bio-Rad). Controls were S. epidermidis strain RP62A (positive control) and S. carnosus TM300 (negative control).

Extracellular cytotoxicity. A549 cells were seeded in 12-well plates two days before infection. Supernatants of bacterial overnight cultures (in TSB, rotational shaking at $\left.37^{\circ} \mathrm{C}\right)$ were sterile-filtered $(0.22 \mu \mathrm{m}$; Millipore, Bedford, MA). A549 cells were incubated with $80 \%$ invasion medium and $20 \%$ filtered supernatant for $24 \mathrm{~h}$. Cells were washed with PBS and adherent cells were detached with EDTA-trypsin. Supernatant, PBS from the washing step and detached cells from one well were collected in one tube, so that cells already detached by cell damage or cell death were included in the analysis. PI-stained cells were detected using flow cytometry. Cytotoxicity was expressed as the proportion of dead (i.e. \% of PI-positive) cells.

Statistics. All statistical analyses were done with "R" applying a significance level of $0.05^{33}$. Continuous variables (e.g. cytotoxicity, invasion, absorption in growth assay) were tested for normal distribution using a quantile-quantile plot (Q-Q plot) with a $45^{\circ}$ reference line and the Shapiro-Wilk test. Outliers and extreme outliers were identified using the package "rstatix". Only if the assumption of normality and homogeneity are met, the two-way repeated measures ANOVA was used to compare the growth curves of S. schweitzeri and S. aureus.

Means of normally distributed continuous variables (i.e. invasion, cytotoxicity, biofilm formation) from $S$. schweitzeri and S. aureus were tested for equivalence with the TOST procedure as implemented in the package "TOSTER". This package includes both a null hypothesis significance test (NHST) and equivalence test. A comparison of the effects measured in the virulence assays between S. schweitzeri and S. aureus (Fig. 3) can remain undetermined if the effects are neither statistically different from zero nor statistically equivalent ${ }^{34}$. We set the equivalence bounds for large effects (Cohen's $\mathrm{d}=0.8)^{35}$.

Where indicated, selected comparisons of continuous variables were done using the two-tailed student's t-test. For individual isolates (see supplement) medians and ranges are reported. 
The datasets generated during and/or analysed during the current study as well as all bacterial strains are available from the corresponding author on reasonable request.

Received: 29 April 2020; Accepted: 24 December 2020

Published online: 13 January 2021

\section{References}

1. Tong, S. Y. et al. Novel staphylococcal species that form part of a Staphylococcus aureus-related complex: The non-pigmented Staphylococcus argenteus sp. nov. and the non-human primate-associated Staphylococcus schweitzeri sp. nov. Int. J. Syst. Evol. Microbiol. 65, 15-22. https://doi.org/10.1099/ijs.0.062752-0 (2015).

2. Becker, K. et al. Implications of identifying the recently defined members of the Staphylococcus aureus complex S. argenteus and S. schweitzeri: A position paper of members of the ESCMID Study Group for Staphylococci and Staphylococcal Diseases (ESGS). Clin. Microbiol. Infect. 1, 1. https://doi.org/10.1016/j.cmi.2019.02.028 (2019).

3. Shittu, A. O., Mellmann, A. \& Schaumburg, F. Molecular characterization of Staphylococcus aureus complex from fomites in Nigeria. Infect. Genet. Evol. 85, 104504. https://doi.org/10.1016/j.meegid.2020.104504 (2020).

4. Schaumburg, F. et al. Staphylococcus aureus complex from animals and humans in three remote African regions. Clin. Microbiol. Infect. 21(345), e341-348. https://doi.org/10.1016/j.cmi.2014.12.001 (2015).

5. Ngoa, U. A. et al. Epidemiology and population structure of Staphylococcus aureus in various population groups from a rural and semi urban area in Gabon, Central Africa. Acta Trop. 124, 42-47. https://doi.org/10.1016/j.actatropica.2012.06.005 (2012).

6. Okuda, K. V. et al. Molecular epidemiology of Staphylococcus aureus from Lambarene, Gabon. Eur. J. Clin. Microbiol. Infect. Dis. 35, 1963-1973. https://doi.org/10.1007/s10096-016-2748-z (2016).

7. Johansson, C., Rautelin, H. \& Kaden, R. Staphylococcus argenteus and Staphylococcus schweitzeri are cytotoxic to human cells in vitro due to high expression of alpha-hemolysin Hla. Virulence 10, 502-510. https://doi.org/10.1080/21505594.2019.1620062 (2019).

8. Kiedrowski, M. R. et al. Nuclease modulates biofilm formation in community-associated methicillin-resistant Staphylococcus aureus. PLoS ONE 6, e26714. https://doi.org/10.1371/journal.pone.0026714 (2011).

9. Schaumburg, F. et al. Characterization of a novel thermostable nuclease homolog (NucM) in a highly divergent Staphylococcus aureus clade. J. Clin. Microbiol. 52, 4036-4038. https://doi.org/10.1128/JCM.02327-14 (2014).

10. Calvignac-Spencer, S., Leendertz, S. A., Gillespie, T. R. \& Leendertz, F. H. Wild great apes as sentinels and sources of infectious disease. Clin. Microbiol. Infect. 18, 521-527. https://doi.org/10.1111/j.1469-0691.2012.03816.x (2012).

11. Guinane, C. M. et al. Evolutionary genomics of Staphylococcus aureus reveals insight into the origin and molecular basis of ruminant host adaptation. Genome Biol. Evol. https://doi.org/10.1093/gbe/evq031 (2010).

12. Lippi, G., Plebani, M. \& Favaloro, E. J. Interference in coagulation testing: Focus on spurious hemolysis, icterus, and lipemia. Semin. Thromb. Hemost. 39, 258-266. https://doi.org/10.1055/s-0032-1328972 (2013).

13. Kulzer, E. Handbook of Zoology Vol. 3 (Walter de Gruyter, Berlin, 2005).

14. Strobel, M. et al. Post invasion events after infection with Staphylococcus aureus are strongly dependent on both the host cell type and the infecting S. aureus strain. Clin. Microbiol. Infect. 22, 799-809. https://doi.org/10.1016/j.cmi.2016.06.020 (2016).

15. Sinha, B. et al. Heterologously expressed Staphylococcus aureus fibronectin-binding proteins are sufficient for invasion of host cells. Infect. Immun. 68, 6871-6878. https://doi.org/10.1128/iai.68.12.6871-6878.2000 (2000).

16. Bur, S., Preissner, K. T., Herrmann, M. \& Bischoff, M. The Staphylococcus aureus extracellular adherence protein promotes bacterial internalization by keratinocytes independent of fibronectin-binding proteins. J. Investig. Dermatol. 133, 2004-2012. https://doi. org/10.1038/jid.2013.87 (2013).

17. Ruffing, U. et al. Community-associated Staphylococcus aureus from Sub-Saharan Africa and Germany: A cross-sectional geographic correlation study. Sci. Rep. 7, 154. https://doi.org/10.1038/s41598-017-00214-8 (2017).

18. Lâm, T. T. et al. Phagolysosomal integrity is generally maintained after Staphylococcus aureus invasion of nonprofessional phagocytes but is modulated by strain 6850. Infect. Immun. 78, 3392-3403. https://doi.org/10.1128/iai.00012-10 (2010).

19. Moldovan, A. \& Fraunholz, M. J. In or out: Phagosomal escape of Staphylococcus aureus. Cell Microbiol. 21, e12997. https://doi. $\mathrm{org} / 10.1111 / \mathrm{cmi} .12997(2019)$.

20. Alexander, E. H. \& Hudson, M. C. Factors influencing the internalization of Staphylococcus aureus and impacts on the course of infections in humans. Appl. Microbiol. Biotechnol. 56, 361-366. https://doi.org/10.1007/s002530100703 (2001).

21. Alabi, A. S. et al. Retrospective analysis of antimicrobial resistance and bacterial spectrum of infection in Gabon, Central Africa. BMC Infect. Dis. 13, 455. https://doi.org/10.1186/1471-2334-13-455 (2013).

22. Schaumburg, F. et al. Highly divergent Staphylococcus aureus isolates from African non-human primates. Environ. Microbiol. Rep. 4, 141-146. https://doi.org/10.1111/j.1758-2229.2011.00316.x (2012).

23. Akobi, B., Aboderin, O., Sasaki, T. \& Shittu, A. Characterization of Staphylococcus aureus isolates from faecal samples of the Straw-Coloured Fruit Bat (Eidolon helvum) in Obafemi Awolowo University (OAU), Nigeria. BMC Microbiol. 12, 1-8. https://doi. org/10.1186/1471-2180-12-279 (2012).

24. Held, J. et al. Bats are rare reservoirs of Staphylococcus aureus complex in Gabon. Infect. Genet. Evol. 47, 118-120. https://doi. org/10.1016/j.meegid.2016.11.022 (2017).

25. Lebughe, M. et al. The impact of the Staphylococcus aureus virulome on infection in a developing country: A cohort study. Front. Microbiol. https://doi.org/10.3389/fmicb.2017.01662 (2017).

26. Brakstad, O. G., Aasbakk, K. \& Maeland, J. A. Detection of Staphylococcus aureus by polymerase chain reaction amplification of the nuc gene. J. Clin. Microbiol. 30, 1654-1660 (1992).

27. Zhang, D. F. et al. Identification of Staphylococcus argenteus in Eastern China based on a nonribosomal peptide synthetase (NRPS) gene. Future Microbiol. 11, 1113-1121. https://doi.org/10.2217/fmb-2016-0017 (2016).

28. Mellmann, A. et al. Real-time genome sequencing of resistant bacteria provides precision infection control in an institutional setting. J. Clin. Microbiol. 54, 2874-2881. https://doi.org/10.1128/jcm.00790-16 (2016).

29. Leopold, S. R., Goering, R. V., Witten, A., Harmsen, D. \& Mellmann, A. Bacterial whole-genome sequencing revisited: Portable, scalable, and standardized analysis for typing and detection of virulence and antibiotic resistance genes. J. Clin. Microbiol. 52, 2365-2370. https://doi.org/10.1128/JCM.00262-14 (2014).

30. Gründling, A. \& Schneewind, O. Cross-linked peptidoglycan mediates lysostaphin binding to the cell wall envelope of Staphylococcus aureus. J. Bacteriol. 188, 2463-2472. https://doi.org/10.1128/jb.188.7.2463-2472.2006 (2006).

31. Voss, A. et al. Expression of S100A8/A9 in HaCaT keratinocytes alters the rate of cell proliferation and differentiation. FEBS Lett. 585, 440-446. https://doi.org/10.1016/j.febslet.2010.12.037 (2011).

32. Ali, H. et al. Identification of suitable reference genes for gene expression studies using quantitative polymerase chain reaction in lung cancer in vitro. Mol. Med. Rep. 11, 3767-3773. https://doi.org/10.3892/mmr.2015.3159 (2015).

33. R Core Team. R: A language and environment for statistical computing (R Foundation for Statistical Computing, Vienna, Austria, 2019). https://www.R-project.org/. 
34. Lakens, D. Equivalence tests: A practical primer for t tests, correlations, and meta-analyses. Social Psychol. Personality Sci. 8, 355-362. https://doi.org/10.1177/1948550617697177 (2017).

35. Lakens, D. Calculating and reporting effect sizes to facilitate cumulative science: A practical primer for t-tests and ANOVAs. Front. Psychol. https://doi.org/10.3389/fpsyg.2013.00863 (2013).

\section{Acknowledgements}

We thank Beate Schulte, Brigitte Schuhen, Michaela Brück and Carmen Musholt for technical assistance. Vero cells and YFP-CWT expressing A549 cells were kindly provided by Stephan Ludwig (Institute of Molecular Virology, Westfälische Wilhelms-Universität Münster, Germany) and Martin J. Fraunholz (Biocenter, University of Würzburg, Germany) respectively. We gratefully acknowledge two $S$. schweitzeri isolates from Kenichi Okuda (Technical University, Dresden, Germany). The Deutsche Forschungsgemeinschaft (SCHA 1994/5-1) and the Medical Faculty of the University of Münster ("Innovative Medizinische Forschung", IMF, SC121720 and "Mediziner Kolleg" [MedK] fellowship Granted to AG) supported the study. The funders had no role in study design, data collection and interpretation, or the decision to submit the work for publication. We acknowledge support from the Open Access Publication Fund of the University of Muenster.

\section{Author contributions}

F.S. and S.N. conceived and supervised the project and analysed data. A.G. and N.J.F. performed the experiments and analysed data. F.S. and A.M. performed and analysed whole genome sequencing. A.S.A. provided bacterial isolates from Lambaréné (Gabon). A.G., N.J.F., S.N. and F.S. drafted the manuscript. All authors reviewed the manuscript.

\section{Funding}

Open Access funding enabled and organized by Projekt DEAL.

\section{Competing interests}

The authors declare no competing interests.

\section{Additional information}

Supplementary Information The online version contains supplementary material available at https://doi. org/10.1038/s41598-021-80961-x.

Correspondence and requests for materials should be addressed to F.S.

Reprints and permissions information is available at www.nature.com/reprints.

Publisher's note Springer Nature remains neutral with regard to jurisdictional claims in published maps and institutional affiliations.

(c) (i) Open Access This article is licensed under a Creative Commons Attribution 4.0 International License, which permits use, sharing, adaptation, distribution and reproduction in any medium or format, as long as you give appropriate credit to the original author(s) and the source, provide a link to the Creative Commons licence, and indicate if changes were made. The images or other third party material in this article are included in the article's Creative Commons licence, unless indicated otherwise in a credit line to the material. If material is not included in the article's Creative Commons licence and your intended use is not permitted by statutory regulation or exceeds the permitted use, you will need to obtain permission directly from the copyright holder. To view a copy of this licence, visit http://creativecommons.org/licenses/by/4.0/.

(c) The Author(s) 2021 